the various ankylosing spondylarthropathies, and chronic fluorosis, and from the spinal changes in acromegaly, hyperparathyroidism, ochronosis, and simple degenerative and postinfective spondylarthropathies. Being relatively benign and often symptomless, its importance lies essentially in the diagnostic confusion it may cause with these other conditions and in its coexistence in some cases with diabetes mellitus. It is not an uncommon condition, Vernon-Roberts and his colleagues ${ }^{4}$ finding it in 20 of about 500 necropsies.

Recently a study has been reported from the United States of America by Utsinger, Resnick, and Shapiro. ${ }^{5}$ Of their 30 patients 25 were men and 5 women, their ages ranging from 50 to 85 and averaging 67 years. In 23 the main complaint was of stiffness in the thoracolumbar spine of many years' duration, usually mild, intermittent, and non-radiating. This spinal stiffness was most apparent first thing in the morning and faded within an hour with simple movements. Normal spinal flexion, extension, and lateral rotation were present in all patients. Eleven of their patients complained of peripheral bone and joint pain, and in seven this was the main or only complaint, the areas affected being shoulder, hip, knee, and ankle; five had acute synovitis of a single joint. Eight had pain in the heels and in five it was the chief complaint. Laboratory investigations were essentially negative, and no evidence was found of abnormality of serum growth or parathyroid hormones; 132 samples of drinking water consumed by 11 patients showed no fluoride concentration levels above 2 parts per million. Only five patients had fasting hyperglycaemia; three others had gout. Every one of the 30 patients had hyperostosis of the thoracic spine and in 21 it affected the whole spine. A radiolucency was often seen between the newly deposited bone and the underlying vertebral body, and extraspinal manifestations included irregular new bone "whiskering" of the pelvis, large bony spurs, mostly on olecranon and calcaneus, and obvious calcification of sacrotuberous, iliolumbar, and patellar ligaments.

As Forestier and Rotes-Querol ${ }^{1}$ stated in their original paper the mild nature of the symptoms gives little indication for active treatment: the purpose of their publication was to distinguish the condition from ankylosing spondylitis and spinal osteoarthrosis. It is referred to in Hollander and McCarty's textbook on arthritis only in the radiological section ${ }^{6}$; most general textbooks, with a few exceptions, ${ }^{7}$ do not mention the condition. Pathological findings are also scantily reported. The association with diabetes mellitus and obesity has been noted, ${ }^{8-11}$ and Harris and her co-workers ${ }^{12}$ found 4 diabetics in a series of 34 patients with ankylosing hyperostosis. Pain in some region of the back was found in 29 of their cases, and, like Utsinger and his colleagues, ${ }^{5}$ they found peripheral joint complaints not uncommon, 24 of their 34 patients having such symptoms. The diagnosis centres essentially on the spinal radiological findings, but the American workers found the extraspinal features so common that they suggested the title "diffuse idiopathic skeletal hyperostosis." The shorter title suggested by Bywaters and Forestier ${ }^{13}$ at the 6th European Congress of Rheumatology, ankylosing hyperostosis, seems preferable.

Utsinger et al described their recent paper" as "diffuse skeletal abnormalities in Forestier disease"-a title which underlines the debt rheumatology and, indeed, general medicine, owes to this brilliant Frenchman. From his early studies with Sicard on radiographic techniques with iodised oil in the 1920s, his extensive work later on the place of gold salts in the treatment of rheumatoid arthritis, his pioneer research on ankylosing spondylitis, and many other contributions over the years, Jacques Forestier has won the highest place in the esteem and affection of his colleagues. His life history shows that great clinicians can still extend and improve medical knowledge and understanding. Forestier's original description of senile ankylosing hyperostosis of the spine is worth rereading as an example of clarity in the description of clinical and radiological findings.

${ }^{1}$ Forestier, J, and Rotes-Querol, J, Annals of the Rheumatic Diseases, 1950, $9,321$.

2 Forestier, J, Jacqueline, F, and Rotes-Querol, J, Ankylosing Spondylitis, trans A U Desjardins. Springfield, Illinois, Thomas, 1956.

${ }^{3}$ Huskisson, E C, and Hart, F D, foint Disease, 2nd ed, p 9. Bristol, John Wright, 1975.

4 Vernon-Roberts, B, Pirie, C J, and Trenwith, V, Annals of the Rheumatic Diseases, 1974, 33, 281.

5 Utsinger, P D, Resnick, D, and Shapiro, R, Archives of Internal Medicine, 1976, 136, 763.

${ }^{6}$ Martel, W, in Hollander, J L, and McCarty, D J, Arthritis, p 104. Philadelphia, Lea and Febiger, 1972.

${ }^{7}$ Hart, F D, in French's Index of Differential Diagnosis, 10th ed, pp 88, 412, 418. Bristol, John Wright, 1973.

${ }^{8}$ Boulet, P, and Mirouze, J, Annales de Médecine, 1954, 55, 674

${ }^{9}$ Bywaters, E G L, Doyle, F H, and Oakley, N, Arthritis and Rheumatism, 1966, 9, 495.

10 Ott, V R, Perkoval, N, and Regehr, in Reports and Abstracts 6th European Congress of Rheumatology, p 517.

11 Julkunen, H, Heinonen, O P, and Pyörälä, K, Annals of the Rheumatic Diseases, 1971, 30, 605

12 Harris, J, et al, Annals of the Rheumatic Diseases, 1974, 33, 210.

${ }^{13}$ Bywaters, E G L, and Forestier, J, in Reports and Abstracts 6th European Congress of Rheumatology, p 472.

\section{Cannabis psychosis}

Psychotic reactions associated with the use of cannabis may be acute or chronic. As long ago as 1843 O'Shaughnessy $^{1}$ described acute cataleptic reactions among his patients who were prescribed cannabis, and the next year Jacques Moreau ${ }^{2}$ drew attention to hallucinatory phenomena. Since then a series of reports have documented similar and other acute undesirable reactions to cannabis, either self-administered or taken for research purposes. ${ }^{3-10}$ These acute psychotoxic reactions generally last a few hours but very occasionally as long as seven days. The risk of a reaction seems to be dose-related, though novices may experience one from quite small amountsafter smoking a single cigarette. The main features have been described"11 as including "paranoid ideas, illusions, hallucinations, depersonalisation, delusions, confusion, restlessness and excitement. In occasional instances there may be additional features of delirium, disorientation, and marked clouding of consciousness." Severe panic may also be prominent.

While this acute toxic reaction may legitimately be classified as a cannabis psychosis the term is more usually reserved for mental disturbances developing in the setting of long-term use, and there is a great deal of controversy about the part played by the drug. These doubts are partly due to the control the chronic user learns over the effects of the drug but also theoretical and methodological. ${ }^{12}{ }^{13}$ Briefly (and apart from the question of what constitutes psychosis) there is little evidence to suggest that adverse reactions in long-term users occur more often than in other comparable populations. Most reports have paid too little attention to factors other than use of cannabis, such as heredity, environment, personality features, and previous psychiatric history, all of which may affect the vulnerability of the individuals concerned. Furthermore, there does not seem to be any consistent relationship between 
psychosis and dose, frequency, or duration of use of the drug. It is unlikely that one substance can cause such widely different syndromes as hysterical reactions, mania, and schizophrenia; indeed cannabis may not cause any specific disorder (as some claim $^{14}$ ) but may act merely as a non-specific precipitating factor.

Both an amotivational syndrome and organic brain damage are said to occur, but psychosis is said to be the most frequent ill-effect of long term use. Reports of such psychoses span many years and different cultures. ${ }^{14-24}$ Despite the varying quality of the clinical studies the evidence is cumulative. Suspicion is also strengthened by recently acquired knowledge about cannab'noids, the active ingredients. Their lipophilic property implies a cumulative effect on tissues, and the possibility of developing tolerance is a real one. ${ }^{25}{ }^{26}$ Interestingly enough, reports of withdrawal syndromes ${ }^{27} 28$ preceded this knowledge.

Bernhardson and Gunne ${ }^{21}$ in a Swedish study based mainly on case records identified 46 cases of psychosis with a history of cannabis as the dominant drug of use. Fifteen patients aged between 16 and 28 claimed daily consumption of $2-15 \mathrm{~g}$ of cannabis for up to five years. Sixteen had a history of psychosis and 23 had neurotic traits before use of the drug. The onset of illness could be either sudden or gradual, and both episodic and chronic forms were found, with schizophrenia the predominant diagnosis. Seven patients with episodic symptoms relapsed on resumption of the use of cannabis-usually in large amounts and repeatedly. Restlessness, violence or destructiveness, paranoid delusions, and visual or auditory hallucinations dominated the clinical picture in the episodic group, while personality change, thought disorder, and auditory hallucinations were conspicuous among the chronic psychotics-a picture similar to that found in schizophrenics in general.

Of equal interest are two recent reports from India. Varma's ${ }^{22}$ general account of the clinical picture of psychosis was based on a study of 1248 patients seen over a ten-year period, many with several relapses after resumption of smoking cannabis. Again the onset was usually acute, ushered in by a sudden increase in dose, and attacks were episodic. Violence, destructiveness, restlessness, and paranoid features were common symptoms. However, he also repeatedly found disorientation in time, altered levels of consciousness, and vivid and well structured visual hallucinations-features suggesting an organic illness.

Thacore and Shukla ${ }^{23}{ }^{24}$ carried out a controlled comparison of 25 consecutive cases of cannabis psychosis of a paranoid type and a control group suffering from paranoid schizophrenia. The clinically significant differences included a higher frequency of violence, panic, and insight in the cannabis group and schizophrenic thought disorder and apprehension among the controls. Visual hallucinations were found in six patients who smoked charas (a strong preparation of cannabis).

Thacore and Shukla make an interesting suggestion, implied in other data, ${ }^{21} 22$ that "in a prolonged history of heavy cannabis consumption tolerance is developed until a saturation point is reached, further increase leads to decompensation of mental functioning that results in psychosis." This suggests that relapses are not an inevitable consequence of resumption of the use of the drug but are related to using it in quantities which a person cannot tolerate.

These independent reports concur on many aspects of the form of mental disorder seen among chronic and heavy users (periodicity, short duration, precipitation by increased dose, relapse after resumption of use) and about its behavioural aspects-restlessness and a tendency to violence and destructiveness. However, the causal relationship between the drug and the disorder and the nature of the disorder (as a distinct entity) remain open to question. ${ }^{12} 13$ Indeed there is little agreement about the clinical basis of differentiation of cannabis psychosis from functional disorders. Bernhardson and Gunne have emphasised an absence of psychosis in the past, intensity of symptoms, and the degree of violence. Varma has stressed the importance of altered consciousness and vivid visual hallucinations, while Thacore and Shukla emphasised insight and panic.

These questions cannot be answered with the present data. Multicentre clinical studies are needed using agreed criteria of selection and standardised methods of clinical examinations, and research studies of patients identified as suffering from cannabis psychosis, using the drug in a double blind trial with the patient as his own control.

${ }^{1}$ O'Shaughnessy, W B, quoted in Cannabis and Man, eds P H Connell and N Dorn, p 90. Edinburgh, Churchill Livingstone, 1975.

${ }^{2}$ Kalant, Oriana J, International fournal of the Addictions, 1971, 6 (3), 553.

3 Weil, A T, Zinberg, and Nelson, J M, Science, 1968, 162, 1234.

${ }^{4}$ Bromberg, W, American fournal of Psychiatry, 1934, 91, 303.

5 Isbell, H, et al, Psychopharmacologia, 1967, 11, 184.

6 Dally, P, British Medical fournal, 1967, 3, 367.

${ }^{7}$ Hollister, L E, Richards, R K, and Gillespie, H K, Clinical Pharmacology and Therapeutics, 1968, 9, 783.

${ }^{8}$ Weil, A, New England Fournal of Medicine, 1970, 282, 997.

${ }^{9}$ Baker, A A, and Lucas, E G, Lancet, 1969, 1, 148.

10 Talbott, J A, and Teague, J W, fournal of the American Medical Association, 1969, 210, 299.

11 World Health Organisation Technical Report Series No 478, The Use of Cannabis. Geneva, World Health Organisation, 1971.

12 Home Office, appendix 1, p 40. London, HMSO, 1968. (W'ootton Report.)

${ }^{13}$ Rathod, N H, Cannabis and Man, eds P H Connell and N Dorn, p 99. Edinburgh, Churchill Livingstone, 1975.

${ }^{14}$ Kolansky, H, and Moore, W T, fournal of the American Medical Association, 1972, 222, 35.

15 Bromberg, W, American fournal of Psychiatry, 1934, 91, 303.

${ }_{16}$ Chopra, I C, and Chopra, R N, Bulletin of Narcotics, 1957, 9, 4.

${ }_{17}$ Dhunjibhoy, J E, fournal of Mental Science, 1930, 76, 254.

18 Asuni, T, Bulletin of Narcotics, 1964, 16 (2), 171

19 Tennant, F S, and Groesbeck, C J, Archives of General Psychiatry, 1972, 27, 133.

20 Bouquet, R J, Bulletin of Narcotics, 1950, 2 (4), 14.

${ }^{21}$ Bernhardson, G, and Gunne, L M, International fournal of the Addictions, 1972, 7(1), 9 .

22 Varma, L P, Indian fournal of Psychiatry, 1972, 14, 241.

${ }_{23}$ Thacore, V R, and Shukla, S R P, Archives of General Psychiatry, 1976, 33, 383.

24 Thacore, V R, British fournal of Psychiatry, 1973, 123, 225.

25 Paton, W D M, Proceedings of the Royal Society of Medicine, 1973, 66, 718.

${ }^{26}$ Bech, P, Rafaelsen, L, and Rafaelson, O J, Danish Medical Bulletin, 1974, 21, 106.

27 Williams, E G, Himmelbach, C K, and Wikler, A, Public Health Reports, 1946, 61, 1059.

${ }^{28}$ Fraser, J D, Lancet, 1949, 2, 747.

\section{Bronchopleural fistula}

A bronchopleural fistula occurs when an injured bronchus fails to heal. It may be seen after open or closed chest injury, lung abscess, bronchiectasis, or tuberculosis, but of special interest is that type which sometimes complicates lung surgery. Though the size of the injured bronchus is important in determining the symptoms and progress of traumatic or infective fistulas, surgical division and closure of major bronchi may result in a small fistula being recognised many years later. Postoperative bronchopleural fistulas, however, remain a serious threat to the safety of pulmonary resection. The recognised ${ }^{2}$ incidence is low (about $2 \%$ ), but this com- 